\title{
Selection of Ideal Operating Point for Infrared Camera System
}

\author{
Jiř́ Čech, Martin Rozkovec \\ Institute of Information Technologies and Electronics \\ FMMIS, Technical University of Liberec \\ Liberec, Czech Republic \\ jiri.cech@tul.cz, martin.rozkovec@tul.cz
}

\begin{abstract}
The paper presents identification of temperature range and gain parameters of long wave infrared camera. Various characteristics of tunable parameters of microbolometer focal plane array were acquired and the optimal operating point was accordingly selected. The camera temperature characteristic and noise-equivalent temperature difference was measured for selected operating point. Results will be used in a project called robust detection system, where there are special requirements to be met.
\end{abstract}

Keywords-Infrared camera; Long wave infrared range; noise equivalent temperature difference; Uncooled bolometers FPA

\section{INTRODUCTION}

The thermal imaging cameras are used for detection of radiation in infrared part of the electromagnetic spectrum. The infrared (IR) radiation is emitted by all objects with temperature above the absolute zero according to the Planck's law. That allows seeing environment without visible illumination (usable for night vision, surveillance). Emitted energy increases with increasing temperature of observed object and it even passes through colder objects. Such effect is valuable for inspection of electrical wires within walls, thermal isolation of house walls, and various industry products.

The IR camera contains usually one focal plane array (FPA) capable to detect IR radiation. The most advanced IR arrays are made with hybrid complementary metal-oxide-semiconductor (CMOS) architecture. Base on the used material, the FPA's spectral range is selected:

- $\quad$ Near infrared (NIR) range 0.76-1.4 $\mu \mathrm{m}$

- $\quad$ Short wave (SWIR) range $1.4-3 \mu \mathrm{m}$

- $\quad$ Medium wave (MWIR) range 3-8 $\mu \mathrm{m}$

- $\quad$ Long wave (LWIR) range 8-15 $\mu \mathrm{m}$

- $\quad$ Far infrared (FIR) range 15-1000 $\mu \mathrm{m}$

Also the resolution differs from smallest devices operating at $80 \times 80 \mathrm{px}$ up to $1024 \times 1024 \mathrm{px}$, as well as frame rates (Frames per second - FPS), which ranges from tens to hundreds FPS. FPA's with best performance requires special cooling, which allows to achieve higher sensitivity and lower noise equivalent temperature difference (NETD $<50 \mathrm{mK}$ ), but they are more expensive than uncooled FPA's.
The cheap uncooled FPA's are responsible for big boom of using IR cameras in industry. One of the big producers with wide portfolio of FPA's is ULIS [1,5,6,7]. The project of RObust DEtection System (RODES) aims to develop hyperspectral camera for dangerous gases detection. System has two parts, the special hyperspectral lens and LWIR camera. The camera is equipped with uncooled CMOS FPA PICO640 from ULIS, which have $17 \mu \mathrm{m}$ microbolometer, resolution $640 \times 480 \mathrm{px}, 50 \mathrm{FPS}$ and NETD $50 \mathrm{mK}$. We use special analog-digital converting modules to easily connect above mentioned FPA to our processing unit based on an field-programmable gate array (FPGA).

Each FPA requires correction of nonuniformity (NUC) [4] and elimination of defective pixels [3]. The corrections are calculated from calibration on a black body in given range of temperatures based on FPA's settings. Based on the construction, an FPA provides few tunable parameters to change its offset and gain. FPA's are able to tune up to various temperature ranges such as $0-150^{\circ} \mathrm{C}$, $200-500^{\circ} \mathrm{C},-20-200^{\circ} \mathrm{C}$ and even up to $1500^{\circ} \mathrm{C}$.

The architecture [5,7] of selected FPA uses pixel array of active microbolometers with biasing transistors. In the reading cycle's skimming with blind bolometers, biasing transistors and current-voltage conversion is performed. FPA provides five tunable parameters:

- $\quad$ Blind microbolometer biasing (GSK)

- $\quad$ Active microbolometer biasing (GFID,VDET)

- Conversion reference biasing (VBUS)

- Integration time (INT)

With each change of these parameters or FPA's inner temperature the offset and gain of FPA are also changed. This will cause actual correction become invalid and recalibration is required.

The FPA is small complex thermal system and have stable average temperature, which can change through time and use. Because FPA's temperature is greater than absolute zero, the dark current is generated with the absence of the light and cause noise.

\section{IR CAMERA PROPERTIES}

The developed LWIR camera is built in aluminum body, that serves as a heat sink. The dimensions are 
$86 \times 86 \times 54 \mathrm{~mm}$ with special protrusion design of bodywalls. No active cooling is available. For the purpose of the RODES project we need to optimize camera ideally for the range $0-50{ }^{\circ} \mathrm{C}$, where we need to achieve the highest possible sensitivity and the lowest noise.

With Planck's law (1) we computed maximal measured spectral radiance of a blackbody [2] for LWIR spectral range and given temperature range, see Fig.1.

$$
B_{\lambda}(\lambda, T)=\frac{2 h c^{2}}{\lambda^{5}} \frac{1}{e^{\frac{h c}{\lambda k_{B} T}}-1}
$$

Where $B_{\lambda}$ is spectral radiance for given wavelength $\lambda$ and temperature $T$ in Kelvins, $c$ is speed of light, $h$ is Planck constant and $k_{B}$ is Boltzmann constant.

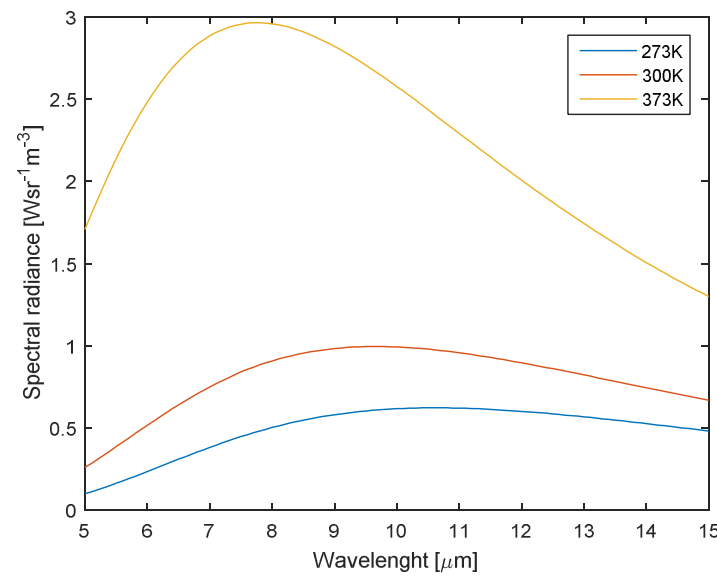

Figure 1. Blackbody spectral radiance for LWIR spectral range

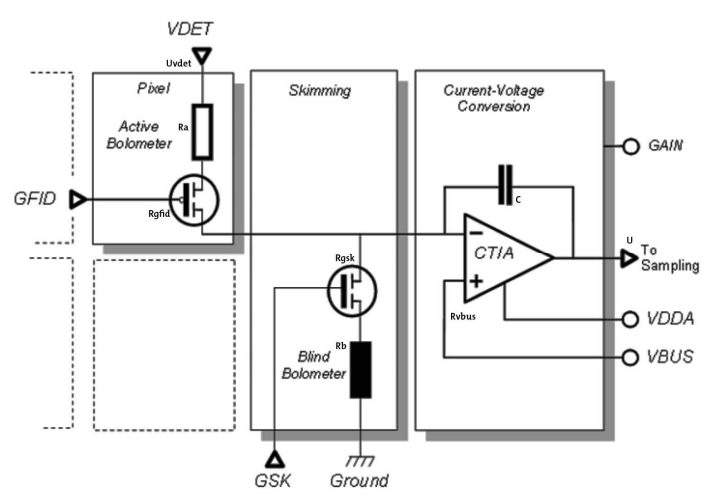

Figure 2. Pixel readout architecture

Each element increases total energy of the circuit proportionally to its resistance. This can be described as (3). Sampling of voltage value $U$ is provided by 16 bit $\mathrm{A} / \mathrm{D}$ convertor and the lowest 2 bits are being removed due to the high noise.

$$
\mathrm{U}=\frac{1}{\mathrm{CR}_{\mathrm{VBUS}}} \int \mathrm{U}_{\mathrm{VDET}} \frac{\mathrm{R}_{\mathrm{GSK}}+\mathrm{R}_{\mathrm{B}}}{\mathrm{R}_{\mathrm{A}}+\mathrm{R}_{\mathrm{GFID}}+\mathrm{R}_{\mathrm{GSK}}+\mathrm{R}_{\mathrm{B}}} d t
$$

Where resistance VBUS is $R_{V B U S}$, GSK is $R_{G S K}$, GFID is $R_{G F I D}$, Active bolometer is $R_{A}$, blind bolometer is $\mathrm{R}_{\mathrm{B}}$, $\mathrm{U}_{\text {VDET }}$ is reference voltage and $C$ is used capacitor. The absorbed voltage is integrated over time INT. We can simplify the equation (2) to (3) for constant INT and camera temperature. Each resistance corresponds to the coefficient $\mathrm{K}$.

$$
\mathrm{U}=\frac{K_{1}}{K_{A}+K_{2}}=\mathrm{K}_{G} * \mathrm{~K}_{A}+\mathrm{K}_{O}
$$

Where coefficient of gain is $K_{G}$, coefficient of offset is $K_{O}$ and coefficient of active bolometer is $K_{A}$, which correspond to detected energy.

$$
\begin{gathered}
K_{1}=\frac{1}{K_{V B U S}} K_{I N T} U_{V D E T}\left(K_{G S K}+K_{B}\right) \\
K_{2}=K_{G F I D}+K_{G S K}+K_{B}
\end{gathered}
$$

The influences of each element is in the gain and also in the offset as it is shown in (6).

$$
\mathrm{K}_{G}=\frac{K_{1}}{K_{A}\left(K_{A}+K_{2}\right)}-\frac{K_{O}}{K_{A}} \quad K_{O}=\frac{K_{1}}{\left(K_{A}+K_{2}\right)}-K_{A} K_{G}
$$

The thermal system of FPA is much more complex and coefficients $K$ are nontrivial functions dependent on the temperature of the FPA and the temperature difference of the scene.

TABLE I. SENSOR ADJUSTABLE PARAMETERS

\begin{tabular}{|c|c|c|c|c|c|}
\hline Parameter & Min & Max & Typical & Selected & Unit \\
\hline VBUS & 1.7 & 3.0 & 2.4 & $\mathbf{2 . 0 5}$ & V \\
\hline VDET & 4.1 & 5.9 & 5.0 & $\mathbf{5 . 0 6}$ & V \\
\hline GSK & 1.9 & 4.4 & 3.3 & $\mathbf{3 . 6 7}$ & V \\
\hline GFID & 0.9 & 3.2 & 1.5 & $\mathbf{0 . 9 1}$ & V \\
\hline INT & 17 & 624 & 624 & 624 & $\begin{array}{c}\text { Clock } \\
\text { Cycles }\end{array}$ \\
\hline
\end{tabular}

The FPA allows changing the gain via value of the capacitor $C$, which multiplies gain by $1 \mathrm{x}, 1.5 \mathrm{x}$ and $3 \mathrm{x}$. We setup it on $3 \mathrm{x}$ gain which correspond to capacitance of $2 \mathrm{pF}$.

\section{IDENTIFICATION}

We measured with blackbody temperatures 10, 20, 30, $40{ }^{\circ} \mathrm{C}$. During each measurement, we have explored complete settings space of the FPA. The raw data from the sensor were acquired with no uniformity correction. The camera temperature was stable with accuracy of $1{ }^{\circ} \mathrm{C}$. The values of $10^{\circ} \mathrm{C}$ blackbody image represents an offset and the gain is calculated for each pixel as difference between $40^{\circ} \mathrm{C}$ blackbody and $10^{\circ} \mathrm{C}$ blackbody, divided by the temperature difference $30^{\circ} \mathrm{C}$.

The measurement for integration time is shown in the Fig. 3, where we can clearly see the linear character of the gain. The high value of the offset for INT 0 is given by the current-voltage conversion offset. The NUC correction eliminates this effect in the images. Changing the INT in this range does have no effect on FPS of FPA. 

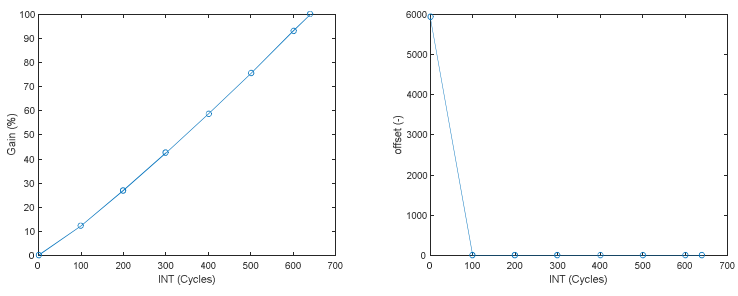

Figure 3. Dependence of gain and offset on INT

Temperature of the FPA affects all properties of the camera [8]. The optimal working temperature of the uncooled FPA is between $20-30^{\circ} \mathrm{C}$, but average working temperature of our camera is $40-50^{\circ} \mathrm{C}$. There is apparent gain increase with temperature shown in the Fig. 4. The increasing FPA's will also increase NETD (from $50 \mathrm{mK}$ up to $2 \mathrm{~K}$ ).
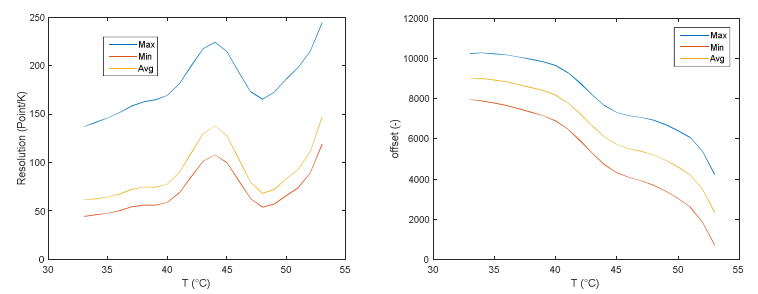

Figure 4. Dependence of gain and offset on Temperature

The reference voltage VDET affects linear gain and offset of pixel as it is shown in the Fig. 5. The typical value is $5 \mathrm{~V}$, but slightly greater values like $5.07 \mathrm{~V}$ lead to a higher gain.
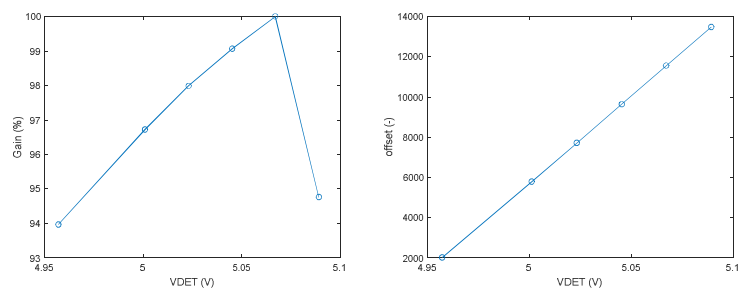

Figure 5. Dependence of gain and offset on VDET

The conversion reference biasing VBUS is available for adjustment only in special series of used convertors. The characteristic shown in the Fig. 6 are not linear and can be different based on actual camera temperature. The typical value is $2.4 \mathrm{~V}$ but our maximum is at $2.16 \mathrm{~V}$.
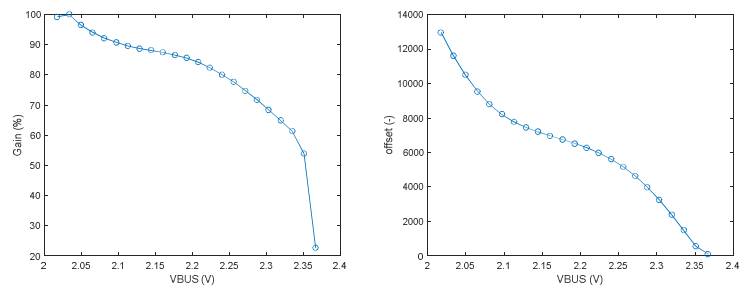

Figure 6. Dependence of gain and offset on VBUS

The main adjustable value that affects gain is GFID. It has linear characteristic as it is shown in the Fig. 7, but it is limited with saturation. There is an inverse effect on FPA's gain and it slightly affects offset, as well.
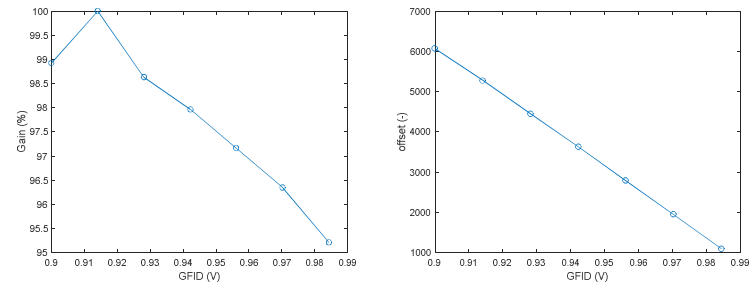

Figure 7. Dependence of gain and offset on GFID

The blind bolometers biasing is main parameter for offset changes in full range of FPA. The gain is slightly affected as it is shown in the Fig. 8.
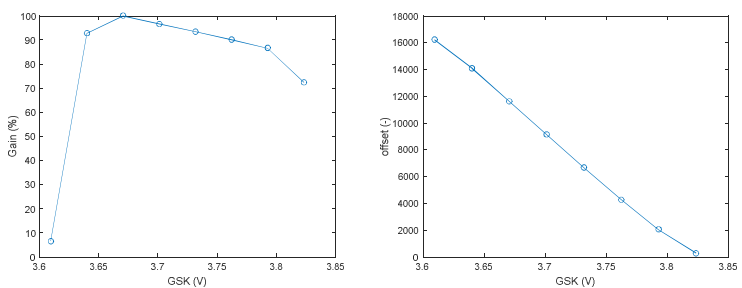

Figure 8. Dependence of gain and offset on GSK

The spectral response of FPA for different wavelengths is shown in the Fig. 9. It is affected by used material (germanium in our case), which have maximal transitivity between $7-15 \mu \mathrm{m}$ and it is used for all lens, we have used.

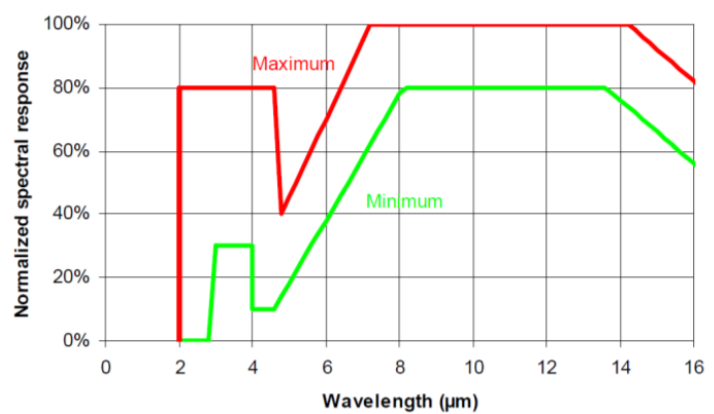

Figure 9. Normalized spectral response of FPA[1]

\section{OPERATING POINT PROPERTIES}

The working point with maximal gain was selected and parameter values are different from the typical in Tab. 1. We achieved measureable temperature range $-20-150^{\circ} \mathrm{C}$ which corresponds to pixel output voltage $4-2.3 \mathrm{~V}$ for camera temperature $43^{\circ} \mathrm{C}$.

The theoretical temperature resolution is $0.1 \mathrm{mV}$, which corresponds to $10 \mathrm{mK}$, but the signal is noisy and the final resolution is given by NETD.

The NETD was measured on 100 samples and computed as standard deviation for blackbody $25^{\circ} \mathrm{C}$ divided by difference of blackbody $30^{\circ} \mathrm{C}$ and $20^{\circ} \mathrm{C}$. The average NETD was $<70 \mathrm{mK}$, but for higher temperature of FPA is NETD greatly increased.

\section{CONCLUSION}

Sensor was adjusted for maximal gain and final temperature range is $-20^{\circ} \mathrm{C}$ to $150^{\circ} \mathrm{C}$, which includes the wanted range $0-50^{\circ} \mathrm{C}$. The resolution is given by 
high NETD 70mK. That are maximal values for given sensor and for achieve better results the sensor replacement is needed. We have in plan use newer version of the same sensor PICO 640 Gen2, which should provide higher FPS and sensitivity.

\section{ACKNOWLEDGMENT}

This work was partially supported by the Student Grant Scheme 2018 of the Technical University of Liberec and by the company APPLIC spol. s. r. o.

\section{REFERENCES}

[1] J.L. Tissot, P. Robert, A. Durand, S. Tinnes, E. Bercier and A. Crastes, "Status of uncooled infrared detector technology at ULIS", France, Defence Science Journal, vol. 63, no. 6, 2013, pp. 545-549.

[2] E. Theocharous, N.P. Fox, V.I. Sapritsky, S.N. Mekhontsev and S.P. Morozova, "Absolute measurements of black-body emitted radiance", Metrologia, vol. 35, no. 4, 1998, pp. 549554.
[3] A.D.R. Girón and H.L. Correa, "A new algorithm for detecting and correcting bad pixels in infrared images", Ingenieria e Investigacion, vol. 30, no. 2, pp. 197-207, 2010.

[4] W. Isoz, T. Svensson and I. Renhorn, "Nonuniformity correction of infrared focal plane arrays", Proceedings of SPIE - The International Society for Optical Engineering, 2005, pp. 949.

[5] T. Sprafke and J. Beletic, "High-Performance Infrared Focal Plane Arrays for Space Applications," Opt. Photon. News 19(6), 2008, pp. 22-27.

[6] K. Gyungtae, K. Heeyeoun, K. Taehyun, S. Jindeok, S. Changho, K. Hyoungho, "High-uniformity post-CMOS uncooled microbolometer focal plane array integrated with active matrix circuit", Sensors and Actuators A: Physical, Volume 211, Pages 138-144, ISSN 0924-4247, 2014.

[7] Y. Park, J. Yun, D. Park, S. Kim and S. Kim, "An Uncooled Microbolometer Infrared Imager With a Shutter-Based Successive-Approximation Calibration Loop," in IEEE Transactions on Very Large Scale Integration (VLSI) Systems, vol. 26, no. 1, 2018, pp. 122-132.

[8] H. Farhat, E. Bazin, S. Haese and G. E. Zein, "An infrared thermal image acquisition system for intra-vehicle applications," 11th International Conference on ITS Telecommunications, St. Petersburg, 2011, pp. 426-430. 